\title{
Activation and inhibition of lymphocytes by costimulation
}

\author{
Kenneth A. Frauwirth and Craig B. Thompson \\ Abramson Family Cancer Research Institute and Department of Cancer Biology, University of Pennsylvania, \\ Philadelphia, Pennsylvania, USA \\ Address correspondence to: Craig B. Thompson, Department of Cancer Biology, University of Pennsylvania, \\ 450 BRB II/III, 421 Curie Boulevard, Philadelphia, Pennsylvania 19104-6160, USA. \\ Phone: (215) 746-5515; Fax: (215) 746-5511; E-mail: drt@mail.med.upenn.edu.
}

J. Clin. Invest. 109:295-299 (2002). DOI:10.1172/JCI200214941.

Mounting an appropriate immune response depends on the careful regulation of lymphocyte activation. To this end, lymphocytes require two independent signals to become fully activated. The first, an antigen-specific signal is sent via the unique antigen receptor: $T$ cell receptor (TCR) on T cells or surface Ig on B cells. The second signal, termed costimulation, is independent of the antigen receptor and is critical to allow full activation, sustain cell proliferation, prevent anergy and/or apoptosis, induce differentiation to effector and memory status, and allow cell-cell cooperation. Costimulation is in turn regulated by the expression of inhibitory receptors upon lymphocyte activation. Here we will consider the growing list of costimulatory and inhibitory molecules, with emphasis on signaling events they initiate. Potentially complex patterns of regulation implied by the variety of receptor/ligand pairs and their differential expression patterns will also be discussed.

\section{What is costimulation?}

It has been recognized for over twenty years that signals in addition to those sent by the antigen receptor are required for full activation of a lymphocyte. Lymphocytes stimulated through the antigen receptor alone fail to produce cytokines, are unable to sustain proliferation, and often undergo apoptosis or become nonresponsive to subsequent stimulation. Early models favored soluble factors as the key transmitters of these signals, and numerous cytokines (IL-1, IL-2, and IL-4, among others) have been found to enhance the activation of both B and T cells. However, it has become clear that interactions between receptor/ligand pairs of cell surface molecules on the responder lymphocyte and an "accessory" cell - an antigen-presenting cell (APC) in the case of $\mathrm{T}$ cell activation, or a helper $\mathrm{T}$ cell for $\mathrm{B}$ cell activation - represent a critical event in the activation process, and it is this event that is generally referred to as costimulation. There is growing evidence for bidirectional communication between the cells, such that a $T$ cell-B cell interaction can involve mutual costimulation and several levels of cross-talk, allowing very specific regulation of lymphocyte activation (Figure 1).
There still appears to be no general agreement on exactly how the term "costimulation" is defined. In some cases it is used broadly to mean nearly any interaction that enhances antigen receptor signaling, while in other cases it is more narrowly construed, meaning only signals that have no stimulatory capacity on their own, but whose synergism with the antigen receptor is required to allow full activation of a naive lymphocyte. In addition, the phrase "negative costimulation" has recently begun to be applied to inhibitory signaling events, further confusing the terminology. For this discussion, costimulation is defined as a signaling pathway that does more than simply augment antigen receptor-proximal activation events, but that intersects with antigen-specific signals synergistically to allow lymphocyte activation. Thus, a costimulatory molecule must initiate a positive signal without simply increasing TCR avidity (as might be the case for adhesion molecules) or enhancing recruitment

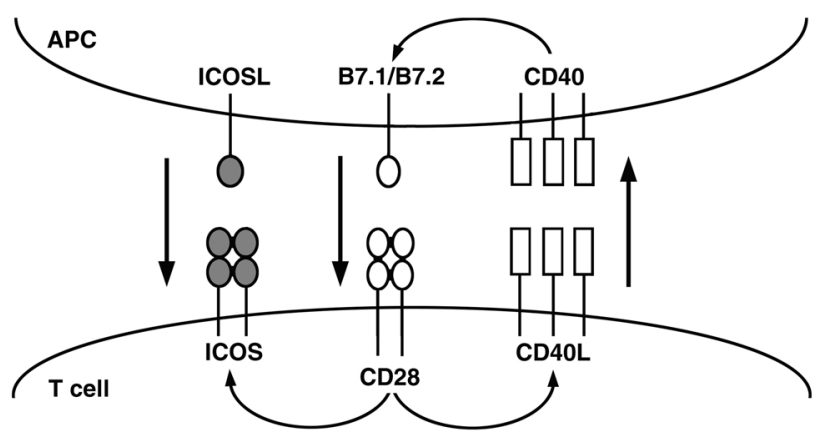

Figure 1

Costimulation involves reciprocal and sequential signals between cells. A T cell-APC interaction begins when the T cell antigen receptor is stimulated by a specific peptide/MHC complex on the surface of the APC (not shown). Low constitutive levels of B7.1 and/or B7.2 on the APC activate $\mathrm{CD} 28$ on the $T$ cell, inducing upregulation of CD40L. CD40 L in turn binds to $C D 40$ on the APC, enhancing B7.1/B7.2 expression and reinforcing the CD28/CD40 positive feedback loop. CD28 costimulation also induces T cell expression of ICOS, allowing a second level of costimulation by APC-expressed ICOSL. Other costimulatory and inhibitory molecules regulated by the initial costimulatory signals (not shown) can further shape the specific outcome of the interaction. 


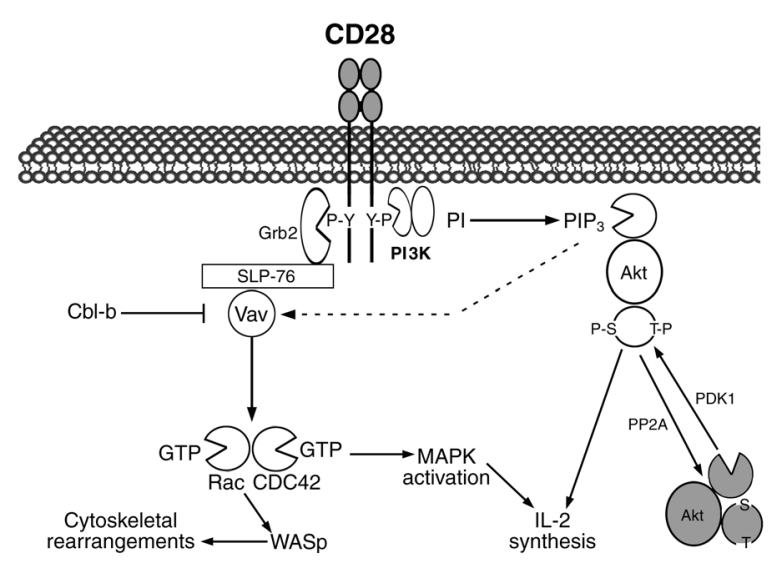

\section{Figure 2}

Pathways implicated in CD28 signaling. Cross-linking of CD28 induces tyrosine phosphorylation of the cytoplasmic tail, allowing interaction with Grb2 and PI3K (although both are portrayed interacting with a single CD28 dimer, it is unknown whether this is a physiological complex). Grb2 links via SLP-76 to the Rho family guanine-nucleotide exchange factor Vav, connecting CD28 to activation of Rac and CDC42. PI3K can signal by recruiting Akt. Negative regulation of Vav by Cbl-b and of Akt by PP2A opposes CD28 costimulatory signals.

of tyrosine kinases to the TCR complex (as is the case for the coreceptors $\mathrm{CD} 4$ and $\mathrm{CD} 8$ ).

\section{Candidate costimulatory molecules}

The first cell surface molecule shown to function as a costimulatory receptor was CD28 (1). Since the identification of CD28, the number of proposed costimulatory molecules has grown significantly $(2,3)$. Most receptors that satisfy the above definition can be divided into two classes based on sequence homologies. The first class contains the related CD28 and inducible costimulator (ICOS) molecules (3). CD28 and ICOS are both disulfide-linked homodimers that bind to distinct members of the B7 family of surface proteins. These appear to be the major costimulatory molecules for the activation of $T$ cells, with naive and resting $T$ cells using CD28 and activated and effector T cells using ICOS. Two other receptors, CTLA-4 and PD-1, share structural homology with this class and also bind B7 family members, but most evidence indicates that both are inhibitory in nature (see below) and thus fail to qualify as costimulatory. These proteins can be considered potential negative regulators of costimulation.

The second class of costimulatory receptors are members of the TNF receptor (TNFR) family. These include CD40, the major B cell costimulatory molecule, as well as OX-40, 4-1BB, CD30, and CD27. The ligands for these receptors are membrane-bound members of the TNF family. Recent reviews have discussed the costimulatory TNFR family members $(2,4)$, so we will focus on advances in understanding the CD28 family of costimulatory molecules.

\section{CD28 signaling}

Studies in vitro and in vivo have shown that CD28 is the primary costimulatory molecule for naive $\mathrm{T}$ cells, although there appears to be differences between $\mathrm{T}$ cell subsets, with $\mathrm{CD}^{+}$("helper") cells more dependent than $\mathrm{CD}^{+}$("cytotoxic") cells on CD28 costimulation in vivo $(4,5)$. However, the signaling pathways downstream of CD28 have been difficult to characterize and have only recently begun to be elucidated (Figure 2). A key event in CD28 signaling appears to be the activation of the small Rho family GTPases Rac and CDC42. Rac and CDC42 activate p21-activated kinase, which may link them to the mitogen-activated protein kinase cascades and the subsequent induction of IL-2 synthesis. Rac and CDC42 are also important in CD28-mediated cytoskeletal rearrangements, through the action of the Wiscott-Aldrich syndrome protein (WASP) (6). However, there is debate regarding the pathways coupling CD28 ligation to these downstream signaling events, with phosphatidylinositol 3-kinase (PI3K), Akt, Vav, and cAMP all receiving recent attention.

\section{PI3K}

PI3K can associate with the cytoplasmic tail of CD28 in a tyrosine phosphorylation-dependent fashion. However, studies using pharmacological inhibitors of PI3K have provided evidence both for and against an essential role for PI3K in CD28 signaling (7). Studies using T cells transfected with CD28 mutants defective for PI3K binding have been similarly equivocal, and even the use of transgenic mice has been unable to resolve this conflict. Two laboratories have recently introduced mutant CD28 constructs unable to bind PI3K into CD28-deficient mice, with strikingly different results. Okkenhaug et al. reported that the mutant $\mathrm{CD} 28$ is indistinguishable from wild-type CD28 in supporting IL-2 synthesis, proliferation, B cell help, and in vivo response to viral infection. Due to a lack of induction of Akt and Bcl-xL, two downstream mediators of CD28 signal transduction, $T$ cells from these animals display increased sensitivity to radiation-induced apoptosis (8). However, Harada et al. reported that $\mathrm{T}$ cells expressing the mutant $\mathrm{CD} 28$ are defective for IL-2 production and proliferation, especially at early time points, and fail to mediate graft-versushost disease (9). It is unclear how such different results arose, since the two groups introduced the same mutant onto essentially identical genetic backgrounds, although they used different promoter/enhancer elements to control CD28 expression. In addition, it has been reported that $\mathrm{T}$ cells from mice lacking the $\mathrm{p} 85 \alpha$ subunit of PI3K appear to function normally (10). Since the p $85 \alpha$ subunit is believed to be required for CD28 binding to PI3K, these results also suggest that PI3K is not required for CD28 signaling. However, there may be some compensation from other $\mathrm{p} 85$ isoforms, which are upregulated in the p $85 \alpha$-deficient cells.

Questions concerning PI3K function in costimulation are not limited to CD28. The cytoplasmic tail of the inducible $\mathrm{T}$ cell costimulatory molecule ICOS also binds PI3K. Recent evidence suggests that PI3K is also important for costimulation of NK cells. Notably, the NK cell transmembrane protein DAP10 binds PI3K, and a receptor complex of DAP10 and the NKG2D molecule acts synergistically with other NK cell receptors to induce cytotoxicity and cytokine secretion in a 
PI3K-dependent fashion (11). Paradoxically, as discussed below, the T cell inhibitory receptor CTLA-4 also recruits $\mathrm{PI} 3 \mathrm{~K}$, suggesting a potential negative function for PI3K. Thus, the role of PI3K in costimulation remains unresolved and offers an important area for continued investigation.

\section{Akt}

Akt (also called protein kinase $\mathrm{B}$, or $\mathrm{PKB}$ ) is a serine/threonine kinase that is recruited to the plasma membrane when its pleckstrin homology domain binds phospholipid products of PI3K. At the membrane, phosphorylation by the kinase phosphoinositidedependent kinase 1 (PDK1) and a second kinase (which may be integrin-linked kinase) stimulates Akt (12), allowing it to phosphorylate downstream targets, such as the inhibitory kinase glycogen synthase kinase $3 \beta$, which is inactivated by Akt. Although Akt has been known for some time to protect cells against numerous apoptotic stimuli, its role in $\mathrm{T}$ cell activation has only recently begun to be appreciated. Antibody cross-linking of CD28 induces Akt phosphorylation and activation, even in the absence of signaling through the TCR complex-associated CD3 proteins $(13,14)$, and this activation is PI3K-dependent. Constitutively active Akt is able to substitute for CD28 signals and stimulate IL-2 production when introduced into mature CD28-deficient $\mathrm{T}$ cells (14). The downstream targets of Akt involved in IL-2 induction are unknown. Surprisingly, Akt does not enhance proliferation of CD28-deficient cells, despite restoration of IL-2 secretion. The expression of the IL-2R $\alpha$ subunit (CD25), which is impaired in CD28-deficient $\mathrm{T}$ cells, has not been examined in mutant cells carrying the constitutive form of Akt. In any case, Akt appears to be important in mediating CD28 signals, but it requires cooperation of other pathways for full costimulation.

Vav
The proto-oncogene Vav acts as a guanine-nucleotide
exchange factor for Rac and CDC42, allowing these
molecules to switch from the inactive GDP-bound state
to the active GTP-bound state (6). Signals downstream
of both CD3 and CD28 can independently induce Vav
phosphorylation and activation, but CD28 is signifi-
cantly more potent and induces more sustained phos-
phorylation. Thus, Vav is a candidate for a direct link
between CD28 and Rac/CDC42-controlled events. Vav
interaction with CD28 is likely mediated by its partici-
pation in a multiprotein complex containing the
adapter proteins SLP-76, LAT, and Grb2, as there is a
Grb2-binding site in the cytoplasmic tail of CD28 (see
Leo et al., this Perspective series, ref. 15 ). Vav may receive
some CD28 signals via PI3K, since Vav activity is regu-
lated in part by the binding of its pleckstrin homology
domain to phospholipid products of PI3K activity (16).
Vav is also subject to negative regulation, and the
RING-finger adapter protein Cbl-b appears to play a
major role. T cells from Cbl-b-deficient mice hyperacti-
vate Vav in response to TCR signals and do not require
CD28 costimulation for activation in vitro or in vivo (17,
18). Thus, CD28 signals appear to be required to over- come Cbl-b inhibition of Vav activation. It is not known how Cbl-b regulates Vav activity, but Cbl-b functions as an E3 ubiquitin ligase (19), suggesting that Cbl-b may control the degradation of a Vav regulatory factor via the ubiquitin-proteasome pathway. However, CD28 still has costimulatory function in Cbl-b-deficient $\mathrm{T}$ cells (17, 18). Thus, as with Akt, regulation of Cbl-b/Vav is at most only a part of costimulatory signaling.

\section{cAMP}

The regulation of cAMP levels is critical for many processes in all cell types and is a feature of signal transduction from multiple receptors, including the TCR. However, elevation of cAMP levels in T lymphocytes is inhibitory, suggesting that inhibition or reversal of cAMP accumulation is required for $\mathrm{T}$ cell activation (20). CD28 costimulation induces expression of a cAMP phosphodiesterase, PDE7, leading to reduced cellular cAMP levels. Blocking PDE7 induction with an antisense oligonucleotide inhibits $\mathrm{T}$ cell proliferation, and this inhibition is reversed by addition of a cAMP analog that blocks downstream cAMP signaling (21). Inhibition by cAMP has been reported to affect nearly every pathway important for lymphocyte activation, allowing CD28 signaling to intersect a wide range of antigen receptor signals through a single effector. Interestingly, increased intracellular cAMP has also been implicated in the induction of $\mathrm{T}$ cell anergy, a nonresponsive state that occurs after $T$ cells are stimulated through TCR/CD3 in the absence of costimulation $(22,23)$. Thus, in addition to providing positive signals, CD28 costimulation may also be critical for the elimination of TCR-initiated negative signals.

\section{ICOS and ICOSL}

Although CD28 is a key costimulatory molecule, it does not account for all costimulatory function in $\mathrm{T}$ cells. Mice lacking CD28 are still able to mount effective responses to some viruses (4), and CD28 deficiency actually enhances diabetes in the nonobese diabetic (NOD) mouse strain (24). Further, production of effector cytokines such as IFN- $\gamma$ and IL- 4 can be stimulated to normal levels by APCs lacking both B7.1 and B7.2 (25, 26). In addition, memory $T$ cells are far less dependent than naive $\mathrm{T}$ cells on CD28/B7 costimulation (27). These results suggest that other costimulatory molecules can compensate for the absence of CD28 signaling.

The recently discovered ICOS molecule has attracted a great deal of attention as one such alternative costimulatory molecule. ICOS is structurally related to CD28 but does not detectably bind to B7.1 or B7.2. Instead, the ligand for ICOS (ICOSL) is a novel B7 family member that has been independently cloned by at least six different groups $(3,28)$. As with CD28, the cytoplasmic tail of ICOS is able to bind the PI3K p85 subunit and is associated with lipid kinase activity (29), although it is unknown whether this is important for ICOS signaling.

ICOS is either absent or expressed at very low levels on naive $T$ cells, but it is upregulated upon stimulation (30), suggesting that its costimulatory role is more important for memory and effector $\mathrm{T}$ cells - precisely the cells that are known to have reduced dependence 
on CD28. Indeed, ICOS costimulation contributes to the production of the effector cytokines IFN- $\gamma$, TNF- $\alpha$, IL-4, IL-5, and IL-10, but little IL-2, and studies with ICOS-deficient mice have shown a critical role for ICOS in $\mathrm{T}$ cell help for Ig class switching and germinal center formation (31-33). APCs show differential regulation of B7.1, B7.2, and ICOSL, which may allow specificity in eliciting responses from naive versus activated (or memory) T cells (34-36). Importantly, ICOSL expression can be induced in fibroblasts in culture, and nonlymphoid tissues in mice, by treatment with the inflammatory agents LPS and TNF- $\alpha$, whereas CD28 ligands are not induced in these same tissues by similar treatment (34). Thus, inflamed peripheral tissues would be able to stimulate antigen-experienced T cells, but unable to stimulate naive T cells, due to the differential regulation of the ligands for CD28 and ICOS.

\section{Negative regulation of costimulation}

The requirement for costimulation allows lymphocyte activation to be strictly regulated by modulating the expression of either member (or both) of the costimulatory receptor/ligand pair. An additional level of regulation is achieved by the expression of inhibitory receptors that can initiate negative signals. The balance between such negative signals and positive signals from antigen receptor and costimulatory molecules apparently sets the threshold for lymphocyte activation. The interaction between costimulatory and inhibitory signals appears to be a critical aspect of the regulation of lymphocyte function.

Perhaps the best-studied inhibitory receptor is CTLA-4. CTLA-4 was the second member of the CD28 family to be identified. Like CD28, it binds to B7.1 and B7.2, although with significantly higher affinity. Resting T cells express little surface CTLA-4, but surface levels are increased upon activation, due to both redistribution of an intracellular pool and increased synthesis. CTLA- 4 inhibits $T$ cell proliferation and IL-2 synthesis in response to stimulation with anti-CD3 and anti-CD28 antibodies. Furthermore, preventing CTLA-4/B7 interactions enhances $T$ cell responses in vitro and in vivo (37). These findings suggest a model in which the dynamic regulation of CTLA-4 and B7 levels is responsible for setting the activation threshold of $\mathrm{T}$ cells. Importantly, CD28 costimulation upregulates CTLA-4 expression, such that costimulation is a self-limiting process. CTLA-4 can also inhibit ICOS signaling (38).

CTLA-4 can interfere with T cell activation by both passive and active mechanisms. When CTLA-4 levels are high, and $\mathrm{B} 7$ levels limiting, the higher affinity of CTLA-4 for B7 allows it to outcompete CD28 for B7 binding, preventing CD28/B7 interactions (39). CTLA-4 can also engage inhibitory signal transduction machinery, including the phosphatases SHP-2 $(40,41)$ and PP2A $(42)$, which may oppose the action of kinases downstream of CD3 and CD28. CTLA-4 ligation has been shown to inhibit activation of extracellular signal-regulated kinase (ERK) and Jun N-terminal kinase (43), both of which are at the ends of kinase cascades. CTLA-4-associated SHP-2 has been proposed to reduce activation-induced phosphorylation of LAT and TCR $\zeta$ (41), although effects on TCR/CD3-proximal events are controversial (43). In addition, most evidence places SHP-2 in positive, rather than negative, signaling pathways (44). PP2A is able to dephosphorylate and deactivate Akt (45), suggesting that it may play a similar role in CTLA-4 inhibition of costimulatory signals. There is also growing evidence that CTLA-4 interacts with other inhibitory pathways, since mutation of the tyrosine residue critical for the SH2-mediated binding of SHP-2 and PP2A does not abolish CTLA-4 function (46-49). Intriguingly, CTLA-4 also interacts with PI3K, further complicating models of PI3K's contribution to lymphocyte activation and inhibition (7).

PD-1 is another inhibitory member of the CD28 family. As with the other CD28 family members, its ligands, PD-L1 (50) and PD-L2 (51), are related to the B7 proteins. PD-1 is expressed on activated B and T cells (52), and engagement of PD-1 has been shown to inhibit $\mathrm{T}$ cell proliferation and cytokine production in response to anti-CD3 and anti-CD28 antibody stimulation $(50,51)$. Further, mice deficient in PD-1 expression develop autoimmune disorders characterized by production of high titers of autoantibodies (53), indicating an important role in the regulation of autoreactive B cells. Unlike CTLA-4, the cytoplasmic tail of PD-1 contains a sequence known as the immunoreceptor tyrosine-based inhibition motif (ITIM; see Billadeau and Leibson, this Perspective series, ref. 54). The ITIM sequence is found in several classes of inhibitory receptors, including the killer inhibitory receptors found on NK cells and Fc $\gamma$ RIIB on B cells, and functions by recruiting $\mathrm{SH} 2$-containing phosphatases (55). There appear to be receptor and cell type specificities in the identity of the phosphatase (SHP-1, SHP-2, or SHIP) recruited by the ITIM motif, and it is unknown which phosphatase is involved in PD-1 function, although, as with CTLA-4, there is some evidence for preferential activation of SHP-2 $(50,51)$.

\section{On the horizon}

Studies over the past several years have revealed a tremendous complexity in the cell-cell interactions regulating lymphocyte activation, with hints of even greater complexity yet. Genome-based searches will likely continue to uncover previously unknown costimulatory and inhibitory receptors and their respective ligands. The recently discovered B7 family member B7-H3 appears to engage a costimulatory molecule that does not correspond to any of the known CD28 family members (56), indicating the presence of at least one additional member. Also, generation of mice lacking both CD28 and CTLA- 4 has produced evidence for a third B7.1/B7.2 receptor (57). The continued analysis of mice lacking one or more costimulatory receptors and/or ligands should help sort out the unique and overlapping roles of these molecules in the positive and negative regulation of immune responses. We expect that greater understanding of the interplay of the various costimulatory and inhibitory signals will lead to important insights into how lymphocyte differentia- 
tion is specified, effector cells are regulated, memory is generated, and tolerance is maintained. In addition, the newly identified receptor/ligand combinations provide targets for immune therapies, with potentially increased specificity. Likewise, elucidation of the specific signaling pathways engaged by these receptors will likely add to the options for therapeutic intervention in immune dysfunctions.

\section{Acknowledgments}

We would like to thank Sunit Talapatra, Wei-Xing Zong, and Jeffrey Rathmell for critical reading of this manuscript, and other members of the Thompson laboratory for helpful discussions.

1. June, C.H., Ledbetter, J.A., Linsley, P.S., and Thompson, C.B. 1990. Role of the CD28 receptor in T-cell activation. Immunol. Today. 11:211-216.

2. Watts, T.H., and DeBenedette, M.A. 1999. T cell co-stimulatory molecules other than CD28. Curr. Opin. Immunol. 11:286-293.

3. Mueller, D.L. 2000. T cells: a proliferation of costimulatory molecules. Curr. Biol. 10:R227-R230.

4. Whitmire, J.K., and Ahmed, R. 2000. Costimulation in antiviral immunity: differential requirements for CD4(+) and CD8(+) T cell responses. Curr. Opin. Immunol. 12:448-455.

5. Lenschow, D.J., Walunas, T.L., and Bluestone, J.A. 1996. CD28/B7 system of T cell costimulation. Annu. Rev. Immunol. 14:233-258.

6. Reif, K., and Cantrell, D.A. 1998. Networking Rho family GTPases in lymphocytes. Immunity. 8:395-401.

7. Hutchcroft, J.E., and Bierer, B.E. 1996. Signaling through CD28/CTLA-4 family receptors: puzzling participation of phosphatidylinositol-3 kinase. J. Immunol. 156:4071-4074.

8. Okkenhaug, K., et al. 2001. A point mutation in CD28 distinguishes proliferative signals from survival signals. Nat. Immunol. 2:325-332.

9. Harada, Y., et al. 2001. Critical requirement for the membrane-proximal cytosolic tyrosine residue for CD28-mediated costimulation in vivo. J. Immunol. 166:3797-3803.

10. Fruman, D.A., et al. 1999. Impaired B cell development and proliferation in absence of phosphoinositide 3-kinase p85alpha. Science. 283:393-397.

11. Lanier, L.L. 2001. On guard: activating NK cell receptors. Nat. Immunol. 2:23-27.

12. Chan, T.O., Rittenhouse, S.E., and Tsichlis, P.N. 1999. AKT/PKB and other D3 phosphoinositide-regulated kinases: kinase activation by phosphoinositide-dependent phosphorylation. Annu. Rev. Biochem. 68:965-1014.

13. Parry, R.V., et al. 1997. Ligation of the T cell co-stimulatory receptor CD28 activates the serine-threonine protein kinase protein kinase B. Eur. J. Immunol. 27:2495-2501.

14. Kane, L.P., Andres, P.G., Howland, K.C., Abbas, A.K., and Weiss, A. 2001. Akt provides the CD28 costimulatory signal for up-regulation of IL-2 and IFN-gamma but not TH2 cytokines. Nat. Immunol. 2:37-44.

15. Leo, A., Wienands, J., Baier, G., Horejsi, V., and Schraven, B. 2002. Adapters in lymphocyte signaling. J. Clin. Invest. 109:301-309. DOI:10.1172/JCI200214942.

16. Cantrell, D. 1998. Lymphocyte signalling: a coordinating role for Vav? Curr. Biol. 8:R535-R538.

17. Bachmaier, K., et al. 2000. Negative regulation of lymphocyte activation and autoimmunity by the molecular adaptor Cbl-b. Nature. 403:211-216.

18. Chiang, Y.J., et al. 2000. Cbl-b regulates the CD28 dependence of T-cell activation. Nature. 403:216-220.

19. Joazeiro, C.A., et al. 1999. The tyrosine kinase negative regulator $\mathrm{c}-\mathrm{Cbl}$ as a RING-type, E2-dependent ubiquitin-protein ligase. Science. 286:309-312.

20. Kammer, G.M. 1988. The adenylate cyclase-cAMP-protein kinase A pathway and regulation of the immune response. Immunol. Today 9:222-229.

21. Li, L., Yee, C., and Beavo, J.A. 1999. CD3- and CD28-dependent induction of PDE7 required for T cell activation. Science. 283:848-851.

22. Cone, R.E., Cochrane, R., Lingenheld, E.G., and Clark, R.B. 1996. Elevation of intracellular cyclic AMP induces an anergic-like state in Th1 clones. Cell. Immunol. 173:246-251.

23. Boussiotis, V.A., et al. 2000. p27kip1 functions as an anergy factor inhibiting interleukin 2 transcription and clonal expansion of alloreactive human and mouse helper T lymphocytes. Nat. Med. 6:290-297.

24. Lenschow, D.J., et al. 1996. CD28/B7 regulation of Th1 and Th2 subsets in the development of autoimmune diabetes. Immunity. 5:285-293.

25. Schweitzer, A.N., and Sharpe, A.H. 1998. Studies using antigen-presenting cells lacking expression of both B7-1 (CD80) and B7-2 (CD86) show distinct requirements for $\mathrm{B} 7$ molecules during priming versus restimulation of Th2 but not Th1 cytokine production. J. Immunol. 161:2762-2771.
26. Chang, T.T., Jabs, C., Sobel, R.A., Kuchroo, V.K., and Sharpe, A.H. 1999. Studies in B7-deficient mice reveal a critical role for B7 costimulation in both induction and effector phases of experimental autoimmune encephalomyelitis. J. Exp. Med. 190:733-740.

27. London, C.A., Lodge, M.P., and Abbas, A.K. 2000. Functional responses and costimulator dependence of memory CD4+ $\mathrm{T}$ cells. J. Immunol. 164:265-272.

28. Coyle, A.J., and Gutierrez-Ramos, J.C. 2001. The expanding B7 superfamily: increasing complexity in costimulatory signals regulating $\mathrm{T}$ cell function. Nat. Immunol. 2:203-209.

29. Coyle, A.J., et al. 2000. The CD28-related molecule ICOS is required for effective T cell-dependent immune responses. Immunity. 13:95-105.

30. Hutloff, A., et al. 1999. ICOS is an inducible T-cell co-stimulator structurally and functionally related to CD28. Nature. 397:263-266.

31. Dong, C., et al. 2001. ICOS co-stimulatory receptor is essential for T-cell activation and function. Nature. 409:97-101.

32. McAdam, A.J., et al. 2001. ICOS is critical for CD40-mediated antibody class switching. Nature. 409:102-105.

33. Tafuri, A., et al. 2001. ICOS is essential for effective T-helper-cell responses. Nature. 409:105-109.

34. Swallow, M.M., Wallin, J.J., and Sha, W.C. 1999. B7h, a novel costimulatory homolog of B7.1 and B7.2, is induced by TNFalpha. Immunity. 11:423-432.

35. Yoshinaga, S.K., et al. 2000. Characterization of a new human B7-related protein: B7RP-1 is the ligand to the co-stimulatory protein ICOS. Int. Immunol. 12:1439-1447.

36. Aicher, A., et al. 2000. Characterization of human inducible costimulator ligand expression and function. J. Immunol. 164:4689-4696.

37. Thompson, C.B., and Allison, J.P. 1997. The emerging role of CTLA-4 as an immune attenuator. Immunity. 7:445-450.

38. Riley, J.L., et al. 2001. ICOS costimulation requires IL-2 and can be prevented by CTLA-4 engagement. J. Immunol. 166:4943-4948.

39. Carreno, B.M., et al. 2000. CTLA-4 (CD152) can inhibit T cell activation by two different mechanisms depending on its level of cell surface expression. J. Immunol. 165:1352-1356.

40. Marengere, L.E., et al. 1996. Regulation of T cell receptor signaling by tyrosine phosphatase SYP association with CTLA-4. Science. 272:1170-1173.

41. Lee, K.M., et al. 1998. Molecular basis of T cell inactivation by CTLA-4. Science. 282:2263-2266.

42. Chuang, E., et al. 2000. The CD28 and CTLA-4 receptors associate with the serine/threonine phosphatase PP2A. Immunity. 13:313-322.

43. Calvo, C.R., Amsen, D., and Kruisbeek, A.M. 1997. Cytotoxic T lymphocyte antigen 4 (CTLA-4) interferes with extracellular signal-regulated kinase (ERK) and Jun NH2-terminal kinase (JNK) activation, but does not affect phosphorylation of $\mathrm{T}$ cell receptor zeta and ZAP70. J. Exp. Med. 186:1645-1653.

44. Van Vactor, D., O'Reilly, A.M., and Neel, B.G. 1998. Genetic analysis of protein tyrosine phosphatases. Curr. Opin. Genet. Dev. 8:112-126.

45. Millward, T.A., Zolnierowicz, S., and Hemmings, B.A. 1999. Regulation of protein kinase cascades by protein phosphatase 2A. Trends Biochem. Sci. 24:186-191.

46. Nakaseko, C., et al. 1999. Cytotoxic T lymphocyte antigen 4 (CTLA-4) engagement delivers an inhibitory signal through the membrane-proximal region in the absence of the tyrosine motif in the cytoplasmic tail. J. Exp. Med. 190:765-774.

47. Cinek, T., Sadra, A., and Imboden, J.B. 2000. Cutting edge: tyrosine-independent transmission of inhibitory signals by CTLA-4. J. Immunol. 164:5-8.

48. Baroja, M.L., et al. 2000. The inhibitory function of CTLA-4 does not require its tyrosine phosphorylation. J. Immunol. 164:49-55.

49. Masteller, E.L., Chuang, E., Mullen, A.C., Reiner, S.L., and Thompson, C.B. 2000. Structural analysis of CTLA-4 function in vivo. J. Immunol. 164:5319-5327.

50. Freeman, G.J., et al. 2000. Engagement of the PD-1 immunoinhibitory receptor by a novel B7 family member leads to negative regulation of lymphocyte activation. J. Exp. Med. 192:1027-1034.

51. Latchman, Y., et al. 2001. PD-L2 is a second ligand for PD-I and inhibits T cell activation. Nat. Immunol. 2:261-268.

52. Agata, Y., et al. 1996. Expression of the PD-1 antigen on the surface of stimulated mouse $\mathrm{T}$ and B lymphocytes. Int. Immunol. 8:765-772.

53. Nishimura, H., Nose, M., Hiai, H., Minato, N., and Honjo, T. 1999. Development of lupus-like autoimmune diseases by disruption of the PD-1 gene encoding an ITIM motif-carrying immunoreceptor. Immunity. 11:141-151.

54. Billadeau, D.D., and Leibson, P.J. 2002. ITAMs versus ITIMs: striking a balance during cell regulation. J. Clin. Invest. 109:161-168. DOI:10.1172/JCI200214843.

55. Vivier, E., and Daeron, M. 1997. Immunoreceptor tyrosine-based inhibition motifs. Immunol. Today. 18:286-291.

56. Chapoval, A.I., et al. 2001. B7-H3: a costimulatory molecule for T cell activation and IFN-gamma production. Nat. Immunol. 2:269-274.

57. Mandelbrot, D.A., et al. 2001. B7-dependent T-cell costimulation in mice lacking CD28 and CTLA4. J. Clin. Invest. 107:881-887. 